\title{
The Use Of Fibre Optic Sensors for Damage Detection and Location in Structural Materials
}

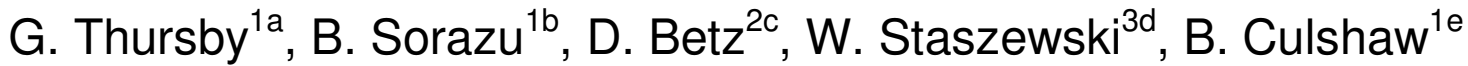 \\ ${ }^{1}$ Optoelectronics, Dept Electronic and Electrical Engineering, University of Strathclyde, 204 George \\ Street, Glasgow G1 1XW \\ 2Daimler Chrysler AG, 89081 Ulm, Germany \\ ${ }^{3}$ Dynamics Research Group, Dept Mechanical Engineering, Sheffield University \\ ag.thursby@eee.strath.ac.uk, bborja@eee.strath.ac.uk' cdaniel.betz@daimlerchrysler.com, \\ dw.j.staszewski@sheffield.ac.uk, ${ }^{\mathrm{e}}$ b.culshaw@eee.strath.ac.uk
}

Keywords: Damage detection, Fibre optics sensors, Ultrasound, Polarimetry, Bragg gratings

\begin{abstract}
The measurement of changes in the properties of ultrasonic Lamb waves propagating through structural material has frequently been proposed as a method for the detection of damage. In this paper we describe work that uses optical fibre sensors to detect the Lamb waves and show that the directional properties of these sensors allow us to not only detect damage, but also to locate it.

We look at two types of optical fibre sensor, a polarimetric sensor and the fibre Bragg grating. The polarimetric sensor measures the change in birefringence of a fibre caused by the pressure wave of the ultrasound acting upon it. This is an integrated sensor since the fibre length bonded to the sample needs to be greater than the ultrasonic wavelength in order to obtain the required sensitivity. The maximum sensitivity of this sensor is when the fibre is positioned normal to the direction of wave propagation. Fibre Bragg gratings are essentially point sensors since the grating length needs to be a fraction of the ultrasound wavelength to obtain maximum sensitivity. Ultrasound is detected mainly through the in-plane strain it produces and maximum sensitivity is therefore produced when the grating is aligned parallel to the direction of wave propagation.

Holes drilled into sample plates can be detected using both type of sensor by examining the changes in either the transmitted Lamb wave or through detection of the reflections produced by the hole. The sensitivity of the technique is shown to be determined by the relative positions of the acoustic source, the hole and the sensor. If we use fibre Bragg gratings in a rosette configuration (i.e. 3 gratings forming an equilateral triangle) then the direction of the Lamb wave can be determined using the directional sensitivities of the gratings. Using two such rosettes allows us to calculate the source of the wave from the intersection of two of these directions. If the source of the wave is the hole (which acts as a passive source), then the location of that hole can be determined.
\end{abstract}

\section{Introduction}

Ultrasound has been used for many years in the field of damage detection in structural materials. The most common technique is to use a wave propagating through the thickness of the material (i.e. normal to the surface), which is an effective technique, but suffers from the drawback that it is very localised and thus requires time-consuming scanning. The use of a guided acoustic Lamb wave that propagates in the plane of the material offers the possibility of interrogating a much larger area with one stationary acoustic source, which results in a reduction in scanning time. Detection of these Lamb waves can be achieved either by the use of conventional sensors, PZT transducers or with optical fibre sensors. In this paper we will discuss two different types of fibre sensor - a polarimetric sensor and the fibre Bragg grating. In both cases the optical fibre is either bonded onto the surface, or embedded within, the material to be interrogated. 


\section{Use of Optical Fibre Sensors for Damage Detection}

Interrogating a structure for potential damage using ultrasound means that we have to compare the acoustic signature of the structure in the damaged state with signals previously obtained from it in the healthy condition. There are two basic methods for doing this, one being to look for changes in the transmitted acoustic signal (e.g. amplitude, phase etc) and the other to look for reflections from the damaged region. Unless the damage is large, the magnitude of either the change in signal or of the reflected signal will be small in comparison to the directly detected acoustic wave. It is therefore important to configure the interrogation scheme in such a way as will provide optimum sensitivity. We demonstrate here that we can use the directional properties of the fibre sensors in order to achieve this by minimising the signal derived directly from the interrogating wave. If we produce a suitable matrix of sensors it also becomes possible to determine the location of damage reflection by the use of a suitable genetic algorithm.

Polarimetric Sensor. When an acoustic wave interacts with an optical fibre bonded onto its surface, the fibre is subjected to a pressure field that induces birefringence into the fibre. If light is launched into the fibre, its polarisation state will be modified by this birefringence. Analysis of the light at the output of the fibre enables us to determine the changes in polarisation state, which can then be related to the pressure field. It can be demonstrated that the optimum sensitivity of this sensor will depend on the input state of polarisation and on the orientation of the analyser. Since the acoustic wave can interact along a fibre whose length is much greater than an acoustic wavelength, we have to think of this as an integrating, rather than a point sensor. The pressure exerted upon the fibre at any particular point will depend on the phase of the acoustic wave at that point. This phase may vary considerably along the length of the fibre in a manner that will depend on the relative shapes of the acoustic wavefront and the fibre sensor. The output of the fibre will then be an integrated measurement of all the phase dependent birefringent changes along the sensor length. The advantage of this type of integrating sensor is that it can be used to cover a wide area. The disadvantage is that the integrating effect can lead to some complex periodic sensitivities with respect to sensor length, acoustic wavelength and the angle between the acoustic wave propagation and the sensor [1]. A typical example is shown in Fig. 1a, although the precise shape will depend on both the sensor length and the distance from the source to the sensor, relative to the acoustic wavelength

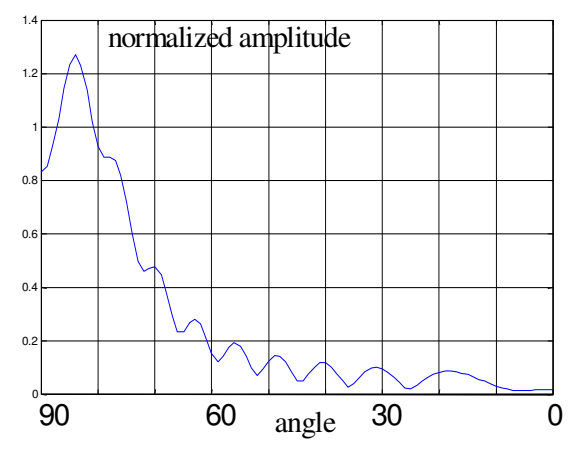

(a) Polarimetric Sensor

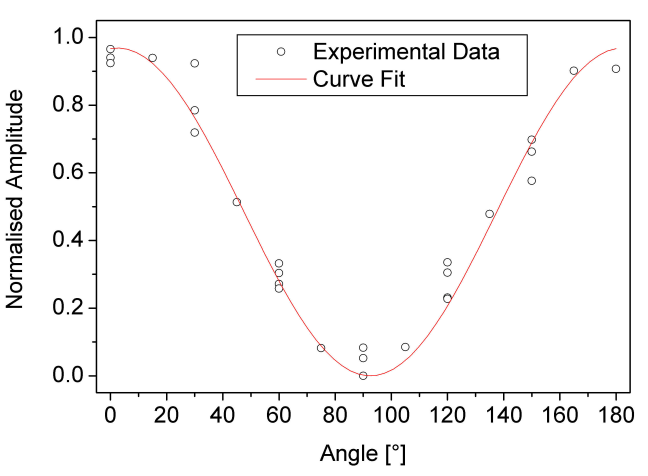

(b) Fibre Bragg Grating

Fig. 1 Comparison of Directional Properties of Fibre Sensors

Fibre Bragg Grating Sensor. A fibre Bragg grating consists of a periodically varying refractive index change that is written into the core of a fibre. Light is reflected at the changes in refractive 
index. The grating will reflect light propagating along the fibre if the optical path length between 2 consecutive reflections is equal to the wavelength of the light, leading to constructive interference between the individual reflections. Straining or changing the temperature of the Bragg grating will result in a change in the wavelength of the reflected light. Bragg gratings can be used to detect ultrasound since Lamb waves produce changing linear strain in the material through which they are travelling [2]. It should also be noted that Lamb waves produce out of plane displacements in addition to linear strain, but that the Bragg gratings are relatively insensitive to these. The length of the Bragg gratings that we have used during our experiments is considerably shorter than the acoustic wavelengths and hence we can think of them as point sensors. Bragg gratings are sensitive to the direction of the acoustic waves being most sensitive when the direction of propagation is along the axis of the fibre and at a minimum when the ultrasound is normal to the fibre. This angular relationship (Fig 1b) is much simpler than for the integrating polarimetric sensor.

\section{Experimental Damage Detection}

Polarimetric Sensor. We first consider the case of a hole in a Perspex sheet being detected using the polarimetric sensor. A PZT transducer bonded to the surface of the Perspex is used to launch Lamb waves into the material. In common with other guided waves Lamb waves exhibit modal properties and in this case the two dominant modes launched are the $\mathrm{S}_{0}$ (fundamental symmetric) and $\mathrm{A}_{0}$ (fundamental antisymmetric modes). They show marked differences in propagation velocity and can therefore by readily temporally separated provided that short tone burst signals are used. In these experiments a 5 cycle tone burst was used at a frequency of $150 \mathrm{kHz}$ on a sheet of $3 \mathrm{~mm}$ thickness.

The layout of the sheet (shown in figure 1) allows us to determine the effect of the relative position of the transducers, hole and optical sensor on the damage detection sensitivity. The signal due to the presence of a hole was detected by storing the signal obtained by the optical sensor with the plate in the undamaged state, producing the hole, reacquiring the signal and subtracting the original signal from it. A signal originating from the interaction of the $S_{0}$ Lamb wave mode with hole is clearly detectable. This process was repeated for each of the transducers in turn. It can be seen that the signal acquired directly from the transducer (i.e. with no hole present) is, as expected, a minimum in the case of the transducer being aligned along the axis of the sensor. Consequently the damage signal/interrogating signal amplitude ratio is a maximum [3].

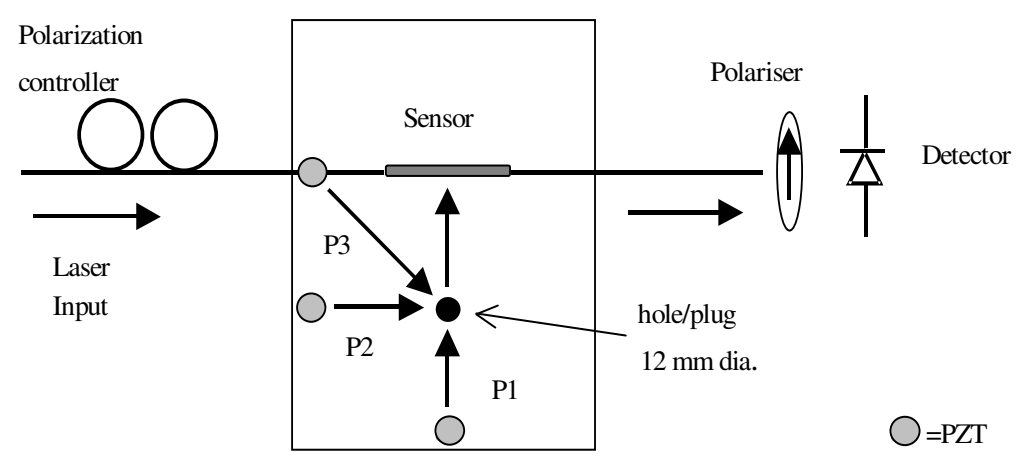

Fig. 2 Experimental layout 


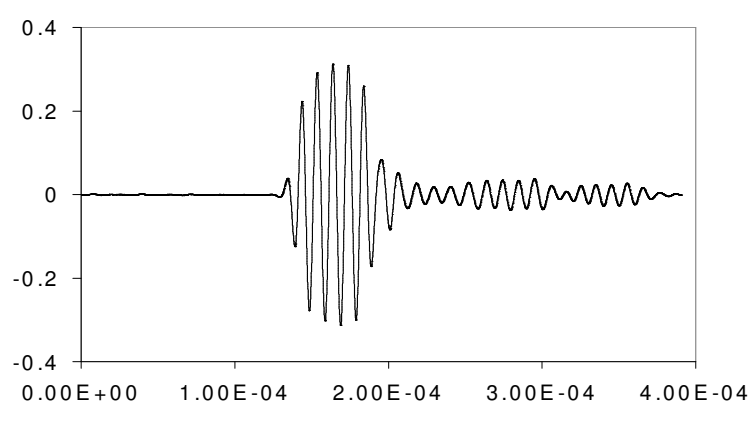

(a)

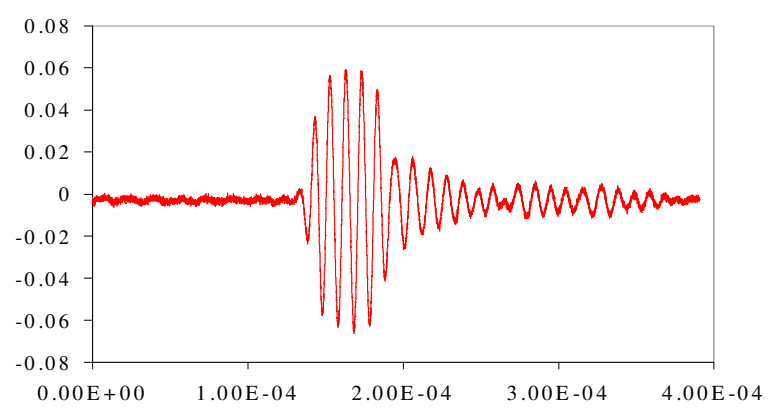

(c)

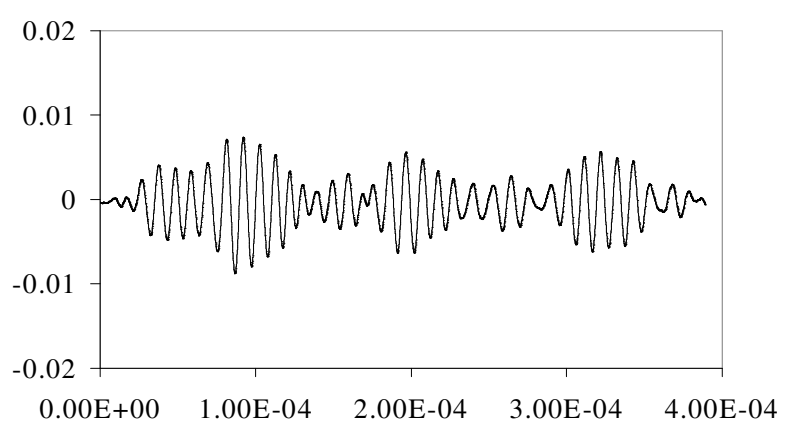

(b)

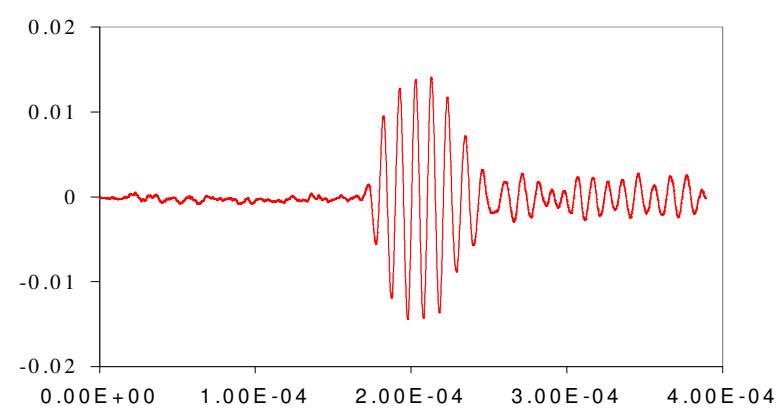

(d)

Fig. 3 (a) Path 1, no hole, (b) Path 3, no hole, (c) Path 1, difference due to hole, (d) Path 3, difference due to hole

Figures 3a-d show the relative size of the signals caused by the holes compared to the original signals, for two different acoustic paths. Note that in path 3 the original signal is small due to the insensitivity of the polarimetric sensor at this angle making the signal due to the hole easier to detect.

Fibre Bragg Gratings. The fibre Bragg gratings were interrogated sequentially using a low noise tuneable laser and measuring the reflected power

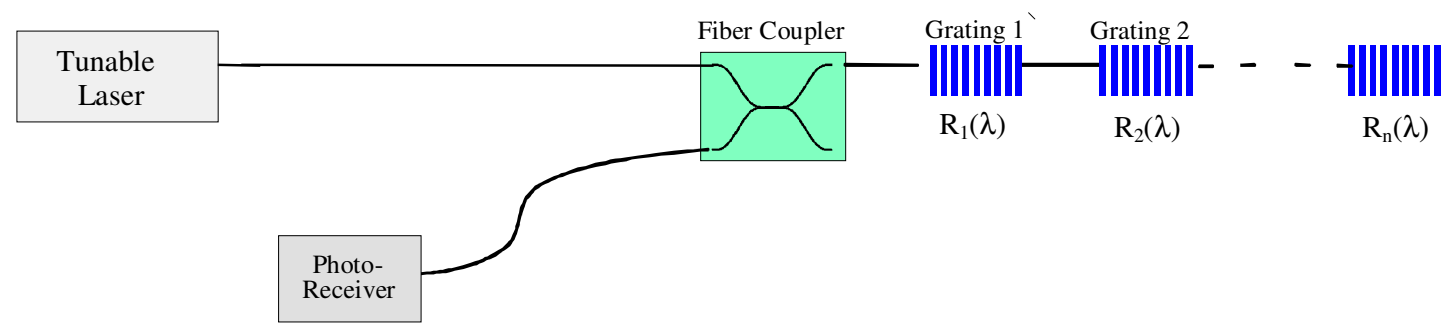

Fig. 4 Interrogation of Bragg gratings

The basic methodology was the same as was used for the polarimetric sensor, but in this case the directional sensitivity of the Bragg gratings was used to determine the location of the hole. Two rosettes of Bragg gratings were bonded to an aluminium plate with the PZT transducers and hole damage located as shown in Fig.5. The direction of propagation of an acoustic wave arriving at a rosette can be determined from the relative amplitudes of the signals obtained from each of the 3 gratings. Calculating the point of intersection of the direction given by the 2 rosettes gives a point, which is the source of the acoustic wave [4]. In order to determine the location of the damage it is 
necessary to measure the response of all 6 Bragg gratings before and after the introduction of the hole and then perform the appropriate subtractions. It is the amplitude of the resultant signals (the reflections from the hole) that must be inserted into the location algorithm. The locations obtained by this method are shown (figure 4) and prove to be remarkably accurate provided that the optimum position of the PZTs relative to the Bragg grating rosettes is used. The letters next to the stars indicate which PZT source was used to obtain that particular location. It is clear that, in order to obtain the most accurate results, the case to be avoided is that in which the source is aligned along the axis of one of the Bragg gratings.

A further benefit of using fibre Bragg gratings for damage detection is that they can also be used for strain field mapping of the structure. The combination of ultrasound damage detection and strain mapping promises to provide a powerful tool for condition monitoring.

Comparison of the Polarimetric and Bragg Grating Sensing Techniques. Although both sensing techniques use optical fibres bonded to the sample plate the two sensing schemes have very different properties. The polarimetric sensor requires the use of standard telecommunications fibre, but needs to be bonded to the sample in such a way that the acoustic pressure field interacts with the fibre in a repeatable, reliable way. The interrogation scheme is very simple, as it requires only a low noise, linearly polarised laser, a polarisation controller to set the optimum polarisation state input for the sensor and an analyser to recover the signal due to the acoustic wave. The sensor can cover a large area for detecting transmitted acoustic waves, but its complex response in terms of the shape of the sensor compared to that of the wavefront may make the results difficult to analyse. Bragg gratings provide a well established method for measuring strain, but in order to detect the extremely small strains produced by an acoustic wave, a very low noise, narrow linewidth, tuneable laser is required to interrogate them. A very sensitive photoreceiver is also needed in order to detect the small changes in light reflected from the gratings. The area covered using directly transmitted waves is clearly smaller than for the polarimetric sensor, but their simple, well-defined directionality means that they may be readily used to detect and locate reflections.

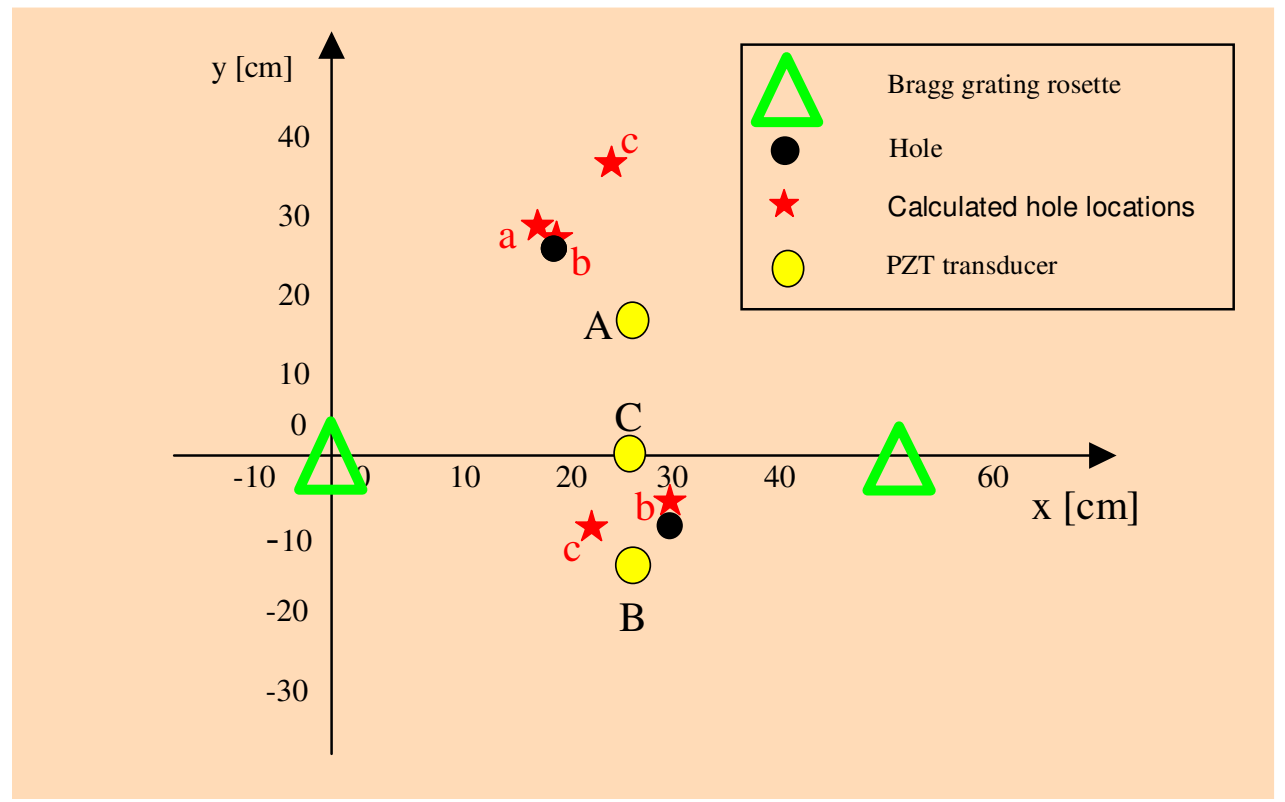

Fig. 5 Layout of sample plate showing comparison between the actual positions of holes and their detected location. 


\section{Conclusions}

Both Bragg gratings and polarimetric sensors can be used to detect ultrasound waves. Bragg gratings are point sensors with a simply defined directionality but require a very stable, low noise tuneable laser to interrogate them. The same sensors may also be used for strain field mapping of a structure. Polarimetric sensors, on the other hand are integrating sensors that require only relatively cheap components to produce an interrogation system. Their main disadvantage may be their relatively complex integration characteristics.

\section{References}

[1] B. Sorazu, G. Thursby, B. Culshaw and F. Dong, OFS-16 Nara, Japan, 2003, p222-5

[2] D.C. Betz, G. Thursby, B. Culshaw and W.J. Staszewski, Smart Mater. Struct., Vol.12, February 2003, p122-128

[3] G. Thursby, B. Sorazu, F. Dong, D. Betz and B. Culshaw, Proc. SPIE Smart Structures and Materials, San Diego, 5050-9, March 2003

[4] D.C. Betz, S. Siedler, G. Thursby, B. Culshaw and W. Staszewski, OFS-16, Nara, Japan, p432-5, October 2003 
Advances in Experimental Mechanics

10.4028/www.scientific.net/AMM.1-2

The Use of Fibre Optic Sensors for Damage Detection and Location in Structural Materials

10.4028/www.scientific.net/AMM.1-2.191

\section{DOI References}

[1] B. Sorazu, G. Thursby, B. Culshaw and F. Dong, OFS-16 Nara, Japan, 2003, p222-5 doi:10.1046/j.1475-1305.2003.00070.x

[2] D.C. Betz, G. Thursby, B. Culshaw and W.J. Staszewski, Smart Mater. Struct., Vol.12, ebruary 2003, p122-128

doi:10.1088/0964-1726/12/1/314 